\title{
Visual mental imagery during caloric vestibular stimulation
}

\author{
Fred W. Mast ${ }^{\mathrm{a}, *}$, Daniel M. Merfeld ${ }^{\mathrm{b}}$, Stephen M. Kosslyn ${ }^{\mathrm{c}}$ \\ ${ }^{\text {a } H a r v a r d ~ U n i v e r s i t y, ~ D e p a r t m e n t ~ o f ~ P s y c h o l o g y, ~ C a m b r i d g e, ~ U S A ~}$ \\ ${ }^{\mathrm{b}}$ Jenks Vestibular Physiology Laboratory, Massachusetts Eye and Ear Infirmary, Harvard Medical School, Boston, USA \\ ${ }^{\mathrm{c}}$ Harvard University, Department of Psychology, Cambridge, USA
}

Received 28 September 2004; received in revised form 17 March 2005; accepted 12 April 2005

Available online 17 May 2005

\begin{abstract}
We investigated high-resolution mental imagery and mental rotation, while the participants received caloric vestibular stimulation. Highresolution visual mental imagery tasks have been shown to activate early visual cortex, which is deactivated by vestibular input. Thus, we predicted that vestibular stimulation would disrupt high-resolution mental imagery; this prediction was confirmed. In addition, mental rotation tasks have been shown to activate posterior parietal cortex, which is also engaged in the processing of vestibular stimulation. As predicted, we also found that mental rotation is impaired during vestibular stimulation. In contrast, such stimulation did not affect performance of a low-imagery control task. These data document previously unsuspected interactions between the vestibular system and the high-level visual system.
\end{abstract}

(C) 2005 Elsevier Ltd. All rights reserved.

Keywords: High-level vision; Occipital cortex; Rotation; Semicircular canal; Sensory interaction

\section{Introduction}

The vestibular system plays a fundamental role in human spatial orientation. For example, it activates the vestibulospinal reflexes needed to control posture and affects gaze via the vestibulo-ocular reflex (VOR). The VOR connects the vestibular end-organ to the eye muscles in such a way that moving the head in one direction induces a compensatory eye movement in the opposite direction. This reflex helps stabilize the image of the world on the retina, when we move our heads. Furthermore, vestibular information is used, when we perceive the orientation of objects (e.g., with respect to gravity, Mittelstaedt, 1983), and it continuously updates the internal representation of space (e.g., Berthoz, Israel, Georges-Francois, Grasso, \& Tsuzuku, 1995).

\footnotetext{
* Corresponding author. Present addresss: University of Zurich, Department of Psychology, Treichlerstrasse 10, CH-8032 Zurich, Switzerland. Tel.: +41 1634 1560; fax: +41 16343132 .

E-mail address: f.mast@psychologie.unizh.ch (F.W. Mast).
}

Numerous neuroimaging studies of the human vestibular system have now been reported, some of which have shown that early visual cortex is deactivated during vestibular stimulation (Bense, Stephan, Yousry, Brandt, \& Dieterich, 2001; Deutschländer et al., 2002; Wenzel et al., 1996). This result is consistent with findings reported by Tiecks, Planck, Haberl, \& Brandt (1996), who, using Doppler sonography, found that vestibular stimulation reduces blood flow in the posterior cerebral artery, which supplies the occipital cortex. Brandt, Bartenstein, Janek, \& Dieterich (1998) and Brandt et al. (2002) interpreted this finding as reflecting an inter-sensory interaction that helps prevent sensory conflicts (e.g., eliminates distracting visual information caused by retinal slip during vestibular stimulation). In fact, researchers have also documented the complementary pattern, a deactivation of vestibular areas during visually induced self-motion (Brandt et al., 1998; Deutschländer et al., 2004) and visual fixation (Naito et al., 2003). These findings are consistent with those from psychophysical studies that show increased thresholds for detecting body acceleration during visually induced body motion (Probst, Straube, \& Bles, 1985). 
The present study took advantage of the finding that vestibular stimulation deactivates the occipital cortex in order to study visual mental imagery. Many neuroimaging studies of visual mental imagery have shown that early visual areas, including area 17, are activated, when people form detailed, high-resolution visual mental images (for review, see Kosslyn \& Thompson, 2003). However, neuroimaging is essentially a correlational technique. Although one transcranial magnetic stimulation study did show that deactivating medial occipital cortex impairs imagery (Kosslyn et al., 1999), this finding does not demonstrate deactivation via natural mechanisms of sensory integration and would, therefore, be more compelling if buttressed by convergent evidence using natural stimulation and entirely different techniques (cf. Pylyshyn, 2002, 2003). If early visual areas are in fact deactivated during vestibular stimulation, and high-resolution visual imagery relies on such areas, then we expect to find impaired performance in high-resolution imagery during such stimulation.

Moreover, we had a second reason for studying mental imagery during vestibular stimulation. Neuroimaging studies have revealed activation in parietal cortex during certain imagery tasks, particularly those involving mental rotation (Alivisatos \& Petrides, 1997; Cohen et al., 1996; Kosslyn, DiGirolamo, Thompson, \& Alpert, 1998; Richter et al., 2000). It is noteworthy that some of the areas found to be engaged in mental rotation tasks are also activated, when people perceive rotation during vestibular stimulation (e.g., the intra-parietal sulcus; Lobel, Kleine, Bihan, Leroy-Willig, \& Berthoz, 1998; Lobel et al., 1999). Therefore, it is likely that such processing may be affected by vestibular stimulation. However, based on previous research, it is possible that simultaneous vestibular stimulation might not interfere, but rather could act to facilitate mental rotation. This alternative is supported by the report that mental imagery deficits following hemispatial neglect can be temporarily attenuated by vestibular stimulation (Rode \& Perenin, 1994; Rode, Perenin, $\&$ Boisson, 1995). This finding suggests that vestibular information is important in constructing a representation of space, and hence vestibular stimulation conceivably could facilitate spatial imagery tasks.

Thus, our goal was to investigate the mechanisms shared by visual mental imagery and vestibular processes. We elicited a relatively constant vestibular response, while participants performed either a high-resolution mental imagery task, a mental rotation task, or a control task that did not require imagery, but instead required participants to evaluate statements about abstract entities. We did not expect vestibular stimulation to affect performance of the control task. In this study, we employed caloric stimulation to elicit vestibular stimulation. Caloric stimulation occurs, when a cold or warm temperature is applied to the outer ear canal, which in turn induces a thermoconvection within the fluid of the horizontal semicircular canal (Barany, 1906; Formby \& Robinson, 2000). This stimulates the horizontal semicircular canal of the vestibular system as if the head were actually rotating.

\section{Methods}

\subsection{Participants}

Eight volunteers participated (five males and three females, ages $24-42$ years) in two separate sessions. They received monetary compensation. Each participant was tested clinically and was verified to have normal vestibular and oculo-motor functions. None of the participants reported any history of vestibular problems or disease. This study was approved by the Institutional Review Boards at the Massachusetts Eye and Ear Infimary and Harvard University.

\subsection{Tasks}

\subsubsection{High-resolution mental imagery}

The participants began by memorizing 40 line drawings of common objects prior to the task (e.g., a grasshopper, a castle or a wrench). These pictures were taken from the standardized set developed by Snodgrass and Vanderwart (1980). The participants were instructed to memorize the objects so that they could later generate vivid mental images of the pictures, the same size as the original with all features. Neuroimaging revealed that early visual areas $17 / 18$ typically are activated, when people visualize these objects (Ganis, Thompson, \& Kosslyn, 2004; Kosslyn, Thompson, Kim, \& Alpert, 1995). During the learning phase, each object was presented separately on a computer screen and the participants had as much time as they needed to memorize it. Once, they felt that they had memorized the object, they pushed a button and the object disappeared. They then formed the mental image of the object they just saw. When the participants had formed the mental image as accurately as possible, they pushed a button and the object reappeared on the screen. They checked whether their image matched the original, visualized again, and corrected the image if necessary. This procedure was repeated twice for each of the 40 pictures. It took the participants roughly $30 \mathrm{~min}$ to complete the learning phase.

During the experiment proper, the participants listened to the names of the objects they studied. As soon as they heard an object's name, they visualized the appropriate object as vividly as possible and retained this image until they heard a cue that indicated a specific judgment. The judgment cues were presented $2 \mathrm{~s}$ after they heard the name of the object. These questions directed the participants to "look" for one of four features on the image. After the learning phase, the participants were familiarized with the four different judgments: (1) Higher than wide: for this judgment, the participants considered the relative width of the imagined object from its leftmost point to its rightmost point (in a straight line), then compared that distance to that from the topmost extreme to the bottommost extreme (again in a straight line). If the distance from top to bottom was greater than the distance from left to right, they were asked to push the Yes-button on a hand-held device. Otherwise, they pushed the No-button. (2) Center higher: for this judgment, the participants considered 
that object's topmost point, and decided whether the topmost point was higher than both the leftmost and rightmost points. They again responded by pushing one of the two buttons. (3) Right higher: the participants judged whether the object's rightmost extreme was higher than its leftmost extreme, and responded accordingly. (4) Enclosed space: the participants judged whether the object had an enclosed space. An object has an enclosed space if there is a space that is completely surrounded by the object but is not part of the object. For example, on a picture of a doughnut, the hole in the middle of the doughnut would be an enclosed space.

\subsubsection{Mental rotation}

The participants saw a set of ten different letters prior to the task. Each letter was presented individually and the participants studied the letter so that they were later able to generate a vivid image, when they heard its name. The letters the participants memorized were either uppercase or lowercase (e.g., b, N, or P). When the participants had memorized the letters, they learned three different cue words, which they heard immediately after the name of the letter during the experiment proper. When they heard the word "right," the participants were to mentally rotate the letter $90^{\circ}$ clockwise and decide whether or not the rotated letter looks like any other uppercase or lowercase upright letter from the alphabet. For example, the letter " $\mathrm{Z}$ " looks like an " $\mathrm{N}$ ", when rotated $90^{\circ}$ clockwise. Correspondingly, they had to rotate the letter $90^{\circ}$ counterclockwise, when they heard the cue word "left," and rotated the letter upside down, when they heard the cue word "down." The participants were specifically instructed to perform all mental rotations in the picture plane. The font of each letter they studied was chosen to ensure that those rotated versions that form a new upright letter were clearly recognized as such. The participants had $4 \mathrm{~s}$ to respond, again pushing one button for "Yes" and another for "No" (using the same response keys as in the high-resolution imagery task).

\subsubsection{Low-imagery task}

We also administered a control task that did not require the participants to use visual mental imagery. The participants verified low-imagery statements by pushing one of two response buttons to indicate whether the statement was "true" or "false" (e.g., "the Portuguese language is more related to Spanish than to German" or "a whale is a fish"). Most of the low-imagery questions were taken from the set developed by Goldenberg et al. (1989) and Goldenberg, Steiner, Podreka, and Deecke (1992). Goldenberg et al. (1992) found lower cerebral blood flow in the left occipital region and higher flow in the right anterior frontal region for these statements compared to high-imagery questions. The high-imagery sentences from Goldenberg et al. (1992) were not used in this study.

\subsubsection{Questionnaire}

We also administered the vividness of visual imagery questionnaire (VVIQ) to assess individual mental imagery ability. This questionnaire consists of descriptions of four familiar scenes, and required the test-taker to visualize four specific aspects of each scene (e.g., a rising sun: the sky clears and surrounds the sun with blueness). The items are rated on a five-point scale, indicating how vivid each image is. The VVIQ has a high-reliability (Marks, 1973) and is relatively unaffected by social-desirability biases (Richardson, 1979).

\subsection{Apparatus}

The participants were tested individually. Each was positioned in a tilt device ("Markham chair") located at the Jenks Vestibular Physiology Laboratory at Massachusetts Eye and Ear Infirmary, and was firmly seated and secured by safety belts. The participant's head was held in place by a head restraint. The seat was suspended in a frame, which could be tilted thus pitching the participant forward and/or backward. The participants wore a blindfold throughout the experiment and all stimuli were presented via loudspeakers attached to the tilt chair. The sound files were prerecorded and presented via Psyscope Software (Cohen, MacWhinney, Flatt, \& Provost, 1993) installed on a Macintosh Powerbook. The participants responded by pushing two buttons on a handheld device. The computer program recorded which button was pushed as well as the response time.

Caloric stimulation was delivered via a closed-loop caloric irrigator manufactured by Brookler Grams. With this irrigator, there was no direct exposure to water, which was circulating in closed-loop tubes. We used bithermal caloric stimulation with $21^{\circ}$ in the right ear and $44^{\circ}$ in the left ear. Stronger stimulation can be elicited with temperatures cooler than $21^{\circ}$ (e.g., with ice water), but we used this temperature because it is much more tolerable for the participants. In order to deliver the stimulation, we inserted a small silicone rubber balloon into each ear canal. The balloon expands, filling the ear canal, as soon as the water pressure builds up as the water circulates through the closed-loop tubes. A properly inflated balloon produces a contact area within the outer ear canal, thus transmitting thermal energy to the mastoid and inner ear. Caloric stimulation can be maintained relatively constant for long periods of time, and can selectively activate the vestibular system. Such constant and selective stimulation would not be possible with protocols using true motion, in which there is always a possibly confounding influence from other sensory sources (e.g., tactile cues due to wind or caused by forces acting on the skin surface during acceleration or deceleration).

Influences from extra-vestibular information also make it difficult to design an appropriate control condition, in which the participants do not experience motion. For caloric stimulation, an adequate control condition consists of applying body temperature to the outer ear canal. This results in sham stimulation, which does not elicit a vestibular response. The thermal energy provided to the mastoid and inner ear is the only difference between caloric and sham caloric stimulation; the rest of the experimental protocol is identical, including the tilted body position, which has been shown to influence 
performance in mental imagery tasks (Mast, Ganis, Christie, \& Kosslyn, 2003).

We also recorded eye movements in order to ensure that nystagmus (a signature of caloric stimulation) was in fact elicited by the caloric irrigation. We did so by attaching electrodes (by adhesive bond) to the outher canthi of the eyes to record horizontal nystagmus, and placed the ground electrode on the forehead. The data were first preamplified, sampled at a rate of $200 \mathrm{~Hz}$ via an analog-to-digital converter using Labview software, and stored in digital format on a hard drive. We calibrated the eye movement recordings by having the participants look at targets displaced $\pm 10^{\circ}$ from center.

\subsection{Protocol}

For this study, we used a novel caloric step stimulus that was described by Formby and Robinson (2000). The stimulus induces a step force to the horizontal semicircular canal by allowing the peripheral thermal dynamics of the caloric transmission to reach steady-state before tilting the participants. The heat flow in the temporal bone reaches a steadystate thermal gradient at about $120 \mathrm{~s}$. Therefore, we applied an initial 2 min period of irrigation, in which the participant was positioned with his/her head pitched forward by $30^{\circ}$. This brings the horizontal semicircular canals roughly into an earth-horizontal plane, such that the canals are not stimulated by thermoconvection. After this phase, the participant was abruptly reclined backward by $90^{\circ}$ (in this final position, the participant is pitched back by $60^{\circ}$ with respect to gravity). Now, the horizontal canal is in an earth-vertical plane, where it is maximally stimulated by caloric stimulation (Barnes, 1995). Caloric irrigation continued during the change in body position and while the participant performed one of the tasks described previously while tilted backwards. Each task was completed within $200 \mathrm{~s}$. Our specific protocol is summarized in Fig. 1.

This reorienting maneuver allows the thermal gradient to induce a step force on the cupula, as if there were a step of angular head acceleration. The step force on the cupula immediately triggers a strong VOR response, which was measured by means of electronystagmography. We used the VOR to ensure that caloric stimulation was properly applied. We knew from clinical screening that each participant showed a normal VOR response during the clinical caloric exam. We used bithermal stimulation to yield binaural vestibular stimulation like that normally elicited by physical body motion.

As soon as the final tilt position was reached, one of the three experimental tasks began. Upon completion of the task, caloric stimulation was switched off and the participant was rapidly moved back to the upright body position. The participants rested for at least $5 \mathrm{~min}$, and we removed the blindfold, while the participant rested in the laboratory (with room lights on). All three tasks were administered in one session, in a counterbalanced order across participants. Each participant completed two separate sessions, one session with binaural caloric stimulation $\left(21^{\circ} \mathrm{C}\right.$ to the right ear, and $44^{\circ} \mathrm{C}$ to the left ear) and one with sham stimulation. We chose to use the right ear for cold stimulation and the left ear for warm stimulation on the basis of a study reported by Shuren, Harley, and Heilman (1998), which showed no neglect-like behavior after rightward real rotation as opposed to leftward real rotation. Despite their use of real physical motion, the binaural vestibular stimulation during motion is similar to the binaural caloric stimulation, we applied in this study. The sham stimulation procedure was identical to caloric stimulation, except that the water was set to normal body temperature $\left(37^{\circ} \mathrm{C}\right)$, and both ears were stimulated the same way. This stimulation does not induce a thermoconvection in the semicircular canals, and therefore, the vestibular system is not stimulated. The order of the test and sham conditions was counterbalanced over participants All eight participants completed both sessions.

\section{Results}

First, we consider the analysis of the error rates (ERs). We conducted a 3 (task type: high-resolution mental imagery, mental rotation, low-mental-imagery sentences) $\times 2$ (stimulation type: sham, caloric) ANOVA with both task type and

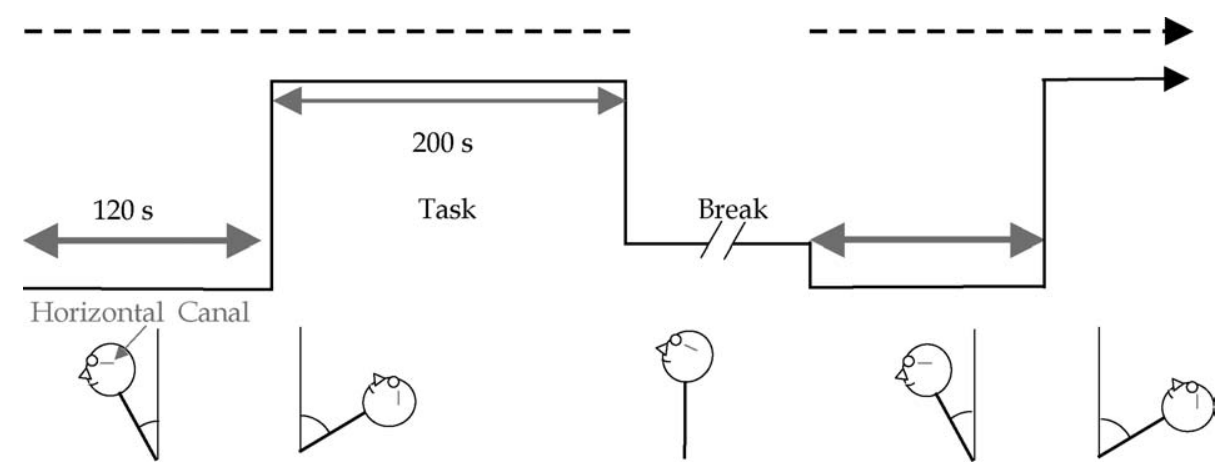

Fig. 1. The caloric step protocol. Caloric stimulation is indicated by the dashed line. The participant is pitched backward by $60^{\circ}$ during the experimental tasks (the horizontal canal is in the earth-vertical position). A period of $120 \mathrm{~s}$ caloric stimulation with the participant pitched forward by $30^{\circ}$ (the horizontal canal is in the earth-horizontal position) preceded each experimental task (see text for details). 


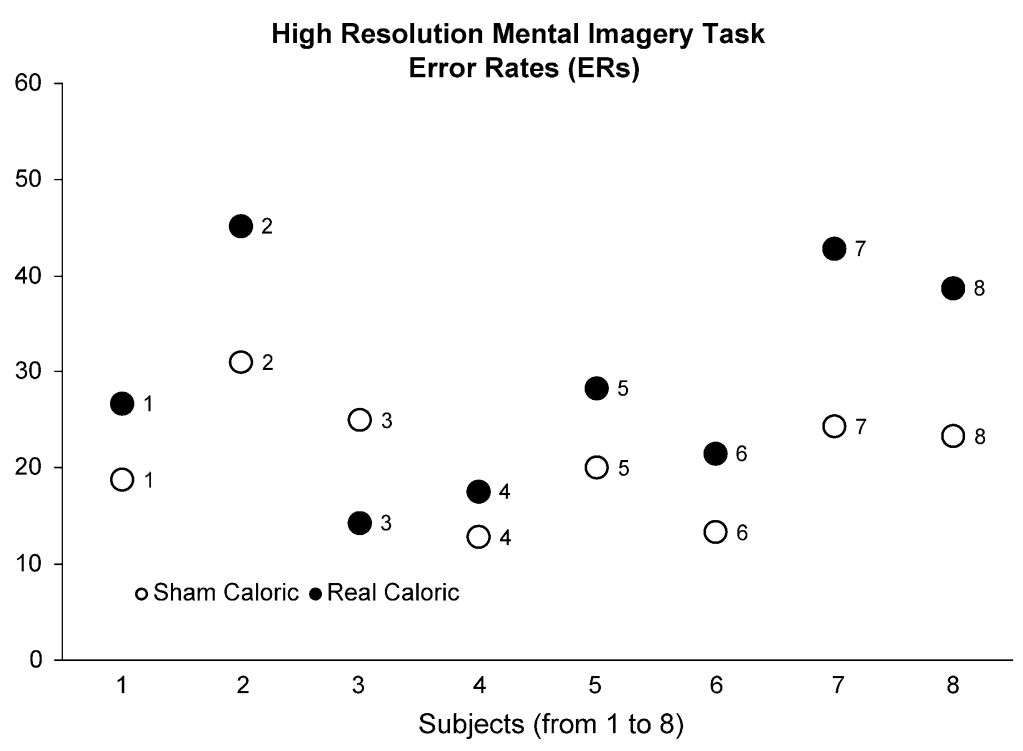

Fig. 2. Error rates for the high-resolution mental imagery task during sham $(\bigcirc)$ and real $(\bullet)$ caloric stimulation shown separately for each participant.

stimulation type as repeated measures and ER as the dependent variable. There was no main effect of task, $F<1$, and no main effect of stimulation per se, $F<1$. Thus, the error rates suggest that the tasks were of comparable difficulty. However, the two variables interacted, $F(2,6)=5.30, p=.047$. Contrast analysis revealed impaired performance, when the participants received caloric stimulation during the high-resolution mental imagery task, $t(7)=6.33, p<.0005$. The mean ERs were $29 \%$ for the caloric stimulation and $21 \%$ for the sham stimulation. Fig. 2 illustrates the ERs for each participant separately. The individual ERs in the high-resolution mental imagery task were higher under caloric stimulation for all but one participant.

In addition, contrast analysis of the ERs revealed that participants were impaired in the mental rotation task, when they received caloric stimulation, $t(7)=11.14, p<.0001$. The participants made $33 \%$ errors under caloric stimulation and $19 \%$ under sham stimulation. Finally, caloric stimulation did not affect performance, while participants evaluated the lowmental-imagery sentences, which served as control; the ERs under caloric $(30 \%)$ and under sham stimulation $(29 \%)$ were virtually identical, $t(7)<1$.

For the analysis of the response times (RTs), we again conducted a 3 (task type: high-resolution mental imagery, mental rotation, low-mental-imagery sentences) $\times 2$ (stimulation type: sham, caloric) ANOVA with both task type and stimulation type as repeated measures and RTs as the dependent variable. We found a significant main effect of task type, $F(2,7)=5.90, p=.038$, indicating that the participants were faster in the mental rotation task $(M=3111 \mathrm{~ms})$ than in the high-resolution mental imagery task $(M=3828 \mathrm{~ms}$, post hoc analyses with Bonferroni adjustment, $p=.011)$. There were no significant differences between RTs in the low-mentalimagery sentences $(M=3332 \mathrm{~ms})$ and the two other tasks. The fact that the control task required about the same amount of time as the two imagery tasks implies that the control task was of comparable difficulty. There was neither an effect of the type of stimulation, $F<1$, nor an interaction between task and stimulation, $F<1$. Note that these findings allow us to rule out the possibility that the effects of caloric stimulation reflect task difficulty per se. The participants responded fastest in the mental rotation task, which was most disrupted in terms of ERs under caloric stimulation. Table 1 summarizes the mean RTs and the mean ERs for all three tasks separately for stimulation type.

Four participants were tested with the caloric stimulation first, followed by the sham stimulation in a separate session, and the other four participants were tested with the sham stim-

Table 1

Mean response times and error rates

\begin{tabular}{|c|c|c|c|c|c|}
\hline & & Mean response time (ms) & Standard error & Mean error rate $(\%)$ & Standard error \\
\hline \multirow[t]{2}{*}{ High-resolution mental imagery } & Caloric & 3854 & 218 & 29 & .04 \\
\hline & Sham & 3803 & 150 & 21 & .02 \\
\hline \multirow[t]{2}{*}{ Mental rotation } & Caloric & 3103 & 195 & 33 & .05 \\
\hline & Sham & 3120 & 280 & 19 & .05 \\
\hline \multirow[t]{2}{*}{ Low-imagery task } & Caloric & 3280 & 114 & 30 & .04 \\
\hline & Sham & 3384 & 159 & 29 & .02 \\
\hline
\end{tabular}


ulation first followed by the caloric stimulation in a separate session. There were no main effects of order revealed by two separate ANOVA's for the mental rotation task, $F(1,6)=1.53$, $p=.26$ for ERs and $F(1,6)=0.23, p=.65$ for RTs, and for the high-resolution mental imagery task, $F(1,6)=1.92, p=.22$ for ERs and $F(1,6)=2.72, p=.15$ for RTs.

We also recorded eye movements during the tasks, which ensured that a caloric nystagmus, a hallmark of vestibular stimulation, was in fact elicited in all participants during caloric stimulation. Visual inspection of the recorded eye movements showed little or no nystagmus during the sham stimulation. Warm water stimulation of the left ear and coldwater stimulation of the right ear caused a caloric nystagmus to the right (direction of slow phase). The mean velocity of the slow phase was $8.2^{\circ} / \mathrm{s}\left(\right.$ S.D. $\left.=5.2^{\circ} / \mathrm{s}\right)$ during the highresolution mental imagery task, $8.4^{\circ} / \mathrm{s}\left(\right.$ S.D. $\left.=5.5^{\circ} / \mathrm{s}\right)$ during the mental rotation task, and $7.5^{\circ} / \mathrm{s}\left(\right.$ S.D. $=5.0^{\circ} / \mathrm{s}$ ) during the low-imagery task. The eye movement data for three out of 24 sessions with caloric stimulation (each of the eight participants completed three tasks) had to be discarded because of technical problems in data acquisition (one session with technical difficulties occurred for each of the three tasks). There was no difference in slow phase velocity during caloric stimulation between the three different types of tasks, as revealed by a one-way ANOVA, $F(2,5)<1$.

We verified that the caloric stimulation was effective by observing the VOR, which was always evident during this condition. Moreover, all participants reported some sense of motion during caloric stimulation, but often they were not able to describe their perception precisely. The stimulation induced a leftward rotation, in terms of vestibular stimulation in the semicircular canals. However, this stimulation conflicts with the direction of gravity, given that the otoliths did not signal a change in orientation with respect to gravity. Therefore, the participants often feel not only rotation, but also translation, which is the result of an internal conflict resolution (as described by Peterka, Gianna-Poulin, Zupan, \& Merfeld, 2004).

Regarding the imagery questionnaire, the mean score for the VVIQ was 54.5 (range: $42-78$, maximum score: 80 ). No significant correlations were found between the VVIQ scores and performance in any of the three tasks, except for the RTs in the mental rotation task, $r(6)=.64, p=.006$.

\section{Discussion}

The results indicate that participants perform two types of mental imagery tasks poorly, when they experience simultaneous vestibular input via caloric stimulation. In contrast, they performed a low-imagery task as well with and without caloric stimulation, which indicates that the observed effects did not reflect overall impaired cognitive performance during caloric stimulation.

Perhaps the most important result of this study is that caloric stimulation impaired performance in the high- resolution imagery task. Previous studies found that caloric stimulation deactivates early visual cortex (Bense et al., 2001; Deutschländer et al., 2004; Tiecks et al., 1996; Wenzel et al., 1996), which led us to predict that such stimulation would impair high-resolution visual mental imagery; numerous studies have shown that high-resolution imagery activates early visual areas (for review, see Kosslyn \& Thompson, 2003). Thus, these findings provide convergent evidence that high-resolution visual mental imagery does in fact rely in part on early visual cortex. The question of whether visual mental imagery is affected by vestibular stimulation was previously addressed primarily in the context of patients with hemispatial neglect (e.g., Guariglia, Padovani, Pantano, \& Pizzamiglio, 1993). Under ordinary conditions, these patients were unable to report any information located in the neglected part of their mental images. However, with caloric stimulation the left-sided neglect was temporarily reduced, both in perception (Cappa, Sterzi, Vallar, \& Bisiach, 1987; Rubens, 1985; Storrie-Baker, Segalowitz, Black, McLean, \& Sullivan, 1997) and imagery (Rode \& Perenin, 1994; Rode, Perenin, \& Boisson, 1995). Even though these findings demonstrate compellingly that caloric vestibular stimulation influences performance in mental imagery, the nature of the neurophysiological mechanisms that underlie the temporary remission of neglect are not yet understood. For example, it is possible that horizontal eye movements elicited during caloric vestibular stimulation could help one to inspect the parts of the image that fall on the otherwise neglected side. Despite the effects shown in neglect patients, only a few studies have been conducted to investigate how caloric vestibular stimulation affects performance in healthy participants (e.g., Karnath, Himmelbach, \& Perenin, 1993). We must note that the present study was not designed to illuminate the mechanisms underlying the temporary remission of the neglect syndrome; our tasks involve primarily object-centered representations whereas vestibular stimulation in unilateral neglect is more likely to manifest itself in space-based coordinates. It is unlikely that the vestibular stimulation we used for this study induced any neglect-like behavior (Shuren et al., 1998); our data do not suggest any tendency in that direction. We were primarily interested in studying mental imagery in the context of a mutually inhibitory relation between visual (including visually imagined) and vestibular information.

However, given our design, we cannot rule out the possibility that our methods induced a conflict between the semicircular canals signalling body rotation and all other cues indicating no change in body position (e.g., sensory information from the otoliths or the participants' knowledge about the stationary body position). None of the participants, however, reported any signs of motion sickness. Moreover, there was no decline in the low-imagery condition, and thus it is unlikely that the caloric stimulation simply distracted the subjects from performing the tasks. What possible mechanisms might be responsible for impaired performance in the high-resolution imagery task? The temporary deactivation of early visual areas during caloric stimulation could 
result from inhibition of remote feedback projections that converge on early visual areas. These feedback projections are massive; indeed, the visual cortex receives roughly the same amount of input from higher areas as it projects to higher areas (e.g., Van Essen, 1985). The functioning of these recurrent connections has been demonstrated in a variety of tasks using attentional selection, perceptual grouping, and visual mental imagery (for review, see Tong, 2003). In the context of this study, no perceptual input was present, and the participants were in complete darkness, when they generated mental images. However, it remains to be determined whether and how the mechanisms of this type of feedback activity are related to the inhibitory inter-sensory mechanism, which leads to a deactivation of early visual areas during vestibular stimulation (Bense et al., 2001; Brandt et al., 1998, 2002; Wenzel et al., 1996).

In this context, it is worth mentioning Kolev's (1995) study, which showed that caloric stimulation evoked visual hallucinations in $67 \%$ of the participants. After the experiment, during verbal debriefing, none of the participants from the present study reported any visual hallucinations, which could have interfered with imagery processing (our specific stimulation paradigm differs from Kolev's, which may explain why we did not find any hallucinations). But even if our participants did experience subtle hallucinations, Kolev (1995) reported that they decayed relatively quickly, after $20 \mathrm{~s}$, a relatively short duration compared to the $200 \mathrm{~s}$ of stimulation, we administered in the present study.

Another important finding of this study was that caloric stimulation impaired performance in a mental rotation task. This task did not require high-resolution imagery (or at least did so to a much lesser extent than the high-resolution imagery task), which suggests that caloric stimulation also affected the mental rotation transformation processes. Behavioral research suggests that at least some processes used in transforming objects in mental images are distinct from those involved in maintaining and inspecting images (Hegarty \& Kozhevnikov, 1999; Mast \& Kosslyn, 2002; Wallace \& Hofelich, 1992). Therefore, it is unlikely that the impaired performance in the mental rotation task had the same origins as the impaired performance in the high-resolution imagery task. The visual cortex is not involved in all types of visual mental imagery, and is typically not drawn upon during mental rotation (Cohen et al., 1996; Thompson \& Kosslyn, 2000). The processes underlying mental rotation are primarily associated with parietal areas (Alivisatos \& Petrides, 1997; Harris \& Miniussi, 2003; Richter et al., 2000), which partly overlap with those areas activated during vestibular stimulation.

In addition, the right hemisphere has sometimes been implicated in mental rotation. For example, several neuropsychological studies report impaired mental rotation of objects in patients with right-sided lesions (Corballis, 1997; Farah \& Hammond, 1998). Furthermore, Harris and Miniussi (2003) applied transcranial magnetic stimulation to the posterior parietal lobe, and only stimulation to the right side affected mental rotation. These findings are interesting in light of re- cent results by Fasold et al. (2002) that show a right-sided dominance during caloric vestibular stimulation, regardless of the stimulated side. However, in light of reports of bilateral activation in several neuroimaging studies of mental rotation, we must be cautious in assuming that only the right hemisphere contributes to such processing (Alivisatos \& Petrides, 1997; Cohen et al., 1996; Thompson \& Kosslyn, 2000).

Although we have focused on early visual cortex and posterior parietal cortex, we must point out that vestibular information is processed in multiple cortical loci. Animal studies have revealed that such information is received by areas in the parietal (area $2 \mathrm{v}$, area $3 \mathrm{aV}$, area 7 , and the parieto-insular vestibular cortex, PIVC), temporal and frontal cortices (area 6pa, area 8a; Grüsser, Pause \& Schreiter, 1982, 1990; Guldin, Akbarian \& Grüsser, 1992; Schwarz \& Fredrickson, 1971). More recently, neuroimaging studies (Bense et al., 2001; Bottini et al., 1994, 2001; Deutschländer et al., 2002; Dieterich et al., 2003; Lobel et al., 1998, 1999; Suzuki et al., 2001; Wenzel et al., 1996) have documented that vestibular stimulation activates the left parieto-temporal junction (e.g., in the posterior insular region, which could be the human analog of the PIVC), the left intra-parietal sulcus and posterior lateral sulcus, and bilaterally activates the central sulcus and the premotor regions of the frontal lobe (BA 44, BA6). Cortical areas with vestibular input were also assessed by electrical stimulation in patients with epilepsy (Kahane, Hoffmann, Minotti, \& Berthoz, 2004). Vestibular sensations could be easily elicited in an area exending above and below the Sylvian fissure (BA 21, BA 22, BA 40).

Thus, in principle, any of these areas (or any combination of these areas) could have contributed to the impaired performance we observed in the two imagery tasks. That said, most of these areas are not typically activated during the imagery tasks we used (Kosslyn \& Thompson, 2003), returning the focus to the early visual cortex and posterior parietal cortex as most likely sites to explain the sensory interaction leading to the observed performance impairment.

The results from this study show that vestibular signals influence two types of visual mental imagery but not another cognitive task. More research is needed to better understand the cortical processing of vestibular information; investigation of the role of vestibular information in cognitive processing is important in order to understand the mechanisms underlying spatial tasks such as those we studied in the domain of visual mental imagery.

\section{Acknowledgments}

We thank Molly Crockett and Dwight Channer for expert technical assistance, and Csilla Haburcakova and Jason Tsajima for their assistance in testing the participants. We are also grateful to William Thompson for providing helpful advice in designing the study and Conrad Wall and the Jenks Vestibular Diagnostic Laboratory for providing the caloric irrigator. This research was supported by NIH/NIDCD Grant 
R01 DC04158 (DMM) and NIH Grant 1 R01 MH60734-01 (SMK), NSF Grant ROLE (SMK), NIMA Grant NMA20101-C-0032 (SMK), and a grant from the Guggenheim Foundation (SMK). The first author was supported by grants from the Swiss National Science Foundation (SNSF 611-066052).

\section{References}

Alivisatos, B., \& Petrides, M. (1997). Functional activation of the human brain during mental rotation. Neuropsychologia, 35, 111-118.

Barany, R. (1906). Untersuchungen über den vom vestibular apparat des Ohres reflektorisch ausgelösten rythmischen Nystagmus und seine Begleiterscheinungen. Monatsschrift für Ohrenheilkunde, 43, 191-236.

Barnes, G. R. (1995). Adaptation in the oculomotor response to caloric irrigation and the merits of bithermal stimulation. British Journal of Audiology, 29, 95-106.

Bense, S., Stephan, T., Yousry, T. A., Brandt, T., \& Dieterich, M. (2001). Multisensory cortical signal increases and decreases during vestibular galvanic stimulation (fMRI). Journal of Neurophysiology, 85, 886-899.

Berthoz, A., Israel, I., Georges-Francois, P., Grasso, R., \& Tsuzuku, T. (1995). Spatial memory of body linear displacement. What is being stored? Science, 269, 95-98.

Bottini, G., Sterzi, R., Paulesu, E., Vallar, G., Cappa, S. F., Erminio, F., et al. (1994). Identification of the central vestibular projections in man: A positron emission tomography activation study. Experimental Brain Research, 99, 164-169.

Bottini, G., Karnath, H.-O., Vallar, G., Sterzi, R., Frith, C. D., Frackowiak, R. S. J., et al. (2001). Cerebral representations for egocentric space: Functional-anatomical evidence from caloric vestibular stimulation and neck vibration. Brain, 124, 1182-1196.

Brandt, T., Bartenstein, P., Janek, A., \& Dieterich, M. (1998). Reciprocal inhibitory visual-vestibular interaction: Visual motion stimulation deactivates the parieto-insular vestibular cortex. Brain, 121, 1749-1758.

Brandt, T., Glasauer, S., Stephan, T., Bense, S., Yousry, T. A., Deutschländer, A., et al. (2002). Visual-vestibular and visuovisual cortical interaction: New insights from fmri and PET. Annals of the New York Academy of Sciences, 956, 230-241.

Cappa, S., Sterzi, R., Vallar, G., \& Bisiach, E. (1987). Remission of hemineglect and anosognosia during vestibular stimulation. Neuropsychologia, 25, 775-782.

Cohen, J. D., MacWhinney, B., Flatt, M., \& Provost, J. (1993). PsyScope: A new graphic interactive environment for designing psychology experiments. Behavior Research Methods, Instruments, and Computers, 25, 257-271.

Cohen, M. S., Kosslyn, S. M., Breiter, H. C., DiGirolamo, G. J., Thompson, W. L., Bookheimer, S. Y., et al. (1996). Changes in cortical activity during mental rotation: A mapping study using functional MRI. Brain, 119, 89-100.

Corballis, M. C. (1997). Mental rotation and the right hemisphere. Brain \& Language, 57, 100-121.

Deutschländer, A., Bense, S., Stephan, T., Schwaiger, M., Brandt, T., \& Dieterich, M. (2002). Sensory system interactions during simultaneous vestibular and visual stimulation in PET. Human Brain Mapping, 16, 92-103.

Deutschländer, A., Bense, S., Stephan, T., Schwaiger, M., Dieterich, M., \& Brandt, T. (2004). Rollvection versus linearvection: Comparison of brain activations in PET. Human Brain Mapping, 21, 143-153.

Dieterich, M., Bense, S., Lutz, S., Drzezga, A., Stephan, T., Bartenstein, P., et al. (2003). Dominance for vestibular cortical function in the non-dominant hemisphere. Cerebral Cortex, 13, 994-1007.

Farah, M. J., \& Hammond, K. M. (1998). Mental rotation and orientationinvariant object recognition: Dissociable processes. Cognition, 29, 29-46.
Fasold, O., von Breveren, M., Kuhberg, M., Ploner, C. J., Villringer, A., Lempert, T., et al. (2002). Human vestibular cortex as identified with caloric stimulation in functional magnetic resonance imaging. Neuroimage, 17, 1384-1393.

Formby, C., \& Robinson, D. A. (2000). Measurement of vestibular ocular reflex (VOR) time constants with a caloric step stimulus. Journal of Vestibular Research, 10, 25-39.

Ganis, G., Thompson, W. L., \& Kosslyn, S. M. (2004). Brain areas underlying visual imagery and visual perception: An fMRI study. Cognitive Brain Research, 20, 226-241.

Goldenberg, G., Podreka, I., Steiner, M., Willmes, K., Süss, E., \& Deecke, L. (1989). Regional cerebral blood flow patterns in visual imagery. Neuropsychologia, 27, 641-664.

Goldenberg, G., Steiner, M., Podreka, I., \& Deecke, L. (1992). Regional cerebral blood flow patterns related to verification of low- and highimagery sentences. Neuropsychologia, 30, 581-586.

Grüsser, O.-J., Pause, M., \& Schreiter, U. (1982). Neuronal responses in the parieto-insular vestibular cortex of alert Java monkeys (Macaca fascicularis). In A. Roucoux \& M. Crommelink (Eds.), Physiological and pathological aspects of eye movements (pp. 25-270). The Haque: W. Junk.

Grüsser, O.-J., Pause, M., \& Schreiter, U. (1990). Localization and responses of neurons in the parieto-insular vestibular cortex of awake monkeys (Macaca fascicularis). Journal of Physiology, 430, 537-557.

Guariglia, C., Padovani, A., Pantano, P., \& Pizzamiglio, L. (1993). Unilateral neglect restricted to visual imagery. Nature, 364, 235-237.

Guldin, W. O., Akbarian, S., \& Grüsser, O.-J. (1992). Cortico-cortical connections and cytoarchitectonics of the primate vestibular cortex: A study in squirrel monkeys (Saimiri sciureus). Journal of Comparative Neurology, 326, 375-401.

Hegarty, M., \& Kozhevnikov, M. (1999). Types of visual-spatial representations and mathematical problem solving. Journal of Educational Psychology, 91, 684-689.

Harris, I. M., \& Miniussi, C. (2003). Parietal lobe contribution to mental rotation demonstrated with rTMS. Journal of Cognitive Neuroscience, $15,315-323$.

Kolev, O. I. (1995). Visual hallucinations evoked by caloric vestibular stimulation in normal humans. Journal of Vestibular Research, 5, 19-23.

Kahane, P., Hoffmann, D., Minotti, L., \& Berthoz, A. (2004). Reappraisal of the human vestibular cortex by cortical electrical stimulation study. Neurology, 63, 122-128.

Karnath, H.-O., Himmelbach, M., \& Perenin, M. T. (2003). Neglect-like behavior in healthy subjects: Dissociation of space exploration and goal-directed pointing after vestibular stimulation. Experimental Brain Research, 153, 231-238.

Kosslyn, S. M., \& Thompson, W. L. (2003). When is early visual cortex activated during visual mental imagery? Theory and meta-analysis. Psychological Bulletin, 129, 723-746.

Kosslyn, S. M., Thompson, W. L., Kim, I. J., \& Alpert, N. M. (1995). Topographical representations of mental images in primary visual cortex. Nature, 378, 496-498.

Kosslyn, S. M., DiGirolamo, G. J., Thompson, W. L., \& Alpert, N. M. (1998). Mental rotation of objects versus hands: Neural mechanisms revealed by positron emission tomography. Psychophysiology, 35, 151-161.

Kosslyn, S. M., Pascual-Leone, A., Felician, O., Camposano, S., Keenan, J. P., Thompson, W. L., et al. (1999). The role of area 17 in visual imagery: Convergent evidence from PET and rTMS. Science, 284, 167-170.

Lobel, E., Kleine, J. F., Leroy-Willig, A., Van de Moortele, P.-F., Le Bihan, D., Grüsser, O.-J., et al. (1999). Cortical areas activated by bilateral galvanic vestibular stimulation. Annals of the New York Academy of Sciences, 871, 313-323.

Lobel, E., Kleine, J. F., Bihan, D. L., Leroy-Willig, A., \& Berthoz, A. (1998). Functional MRI of galvanic vestibular stimulation. Journal of Neurophysiology, 80, 2699-2709. 
Marks, D. F. (1973). Visual mental imagery in the recall of pictures. British Journal of Psychology, 64, 17-24.

Mast, F. W., Ganis, G., Christie, S., \& Kosslyn, S. M. (2003). Four types of mental imagery processing in upright and tilted observers. Cognitive Brain Research, 17, 238-247.

Mast, F. W., \& Kosslyn, S. M. (2002). Visual mental images can be ambiguous: Insights from individual differences in spatial transformation abilities. Cognition, 86, 57-70.

Mittelstaedt, H. (1983). A new solution to the problem of the subjective vertical. Naturwissenschaften, 70, 272-281.

Naito, Y., Tateva, I., Hirano, S., Inoue, M., Funabiki, K., Toyoda, H., et al. (2003). Cortical correlates of vestibulo-ocular reflex modulation: A PET study. Brain, 126, 1562-1578.

Peterka, R. J., Gianna-Poulin, C. C., Zupan, L. H., \& Merfeld, D. M (2004). Origin of orientation-dependent asymmetries in vestibuloocular reflexes evoked by caloric stimulation. Journal of Neurophysiology, 92, 2333-2345.

Probst, T., Straube, A., \& Bles, W. (1985). Differential effects of ambivalent visual-vestibular-somatosensory stimulation on the perception of self-motion. Behavioral Brain Research, 16, 71-79.

Pylyshyn, Z. W. (2002). Mental imagery: In search of a theory. Behavioral \& Brain Sciences, 25, 157-238.

Pylyshyn, Z. (2003). Return of the mental image: Are there pictures in the brain? Trends in Cognitive Sciences, 7, 113-118.

Richardson, A. (1979). Dream recall frequency and vividness of visual imagery. Journal of Mental Imagery, 3, 65-72.

Richter, W., Somorjai, R., Summers, R., Jarmasz, M., Menon, R. S., Gati, J. S., et al. (2000). Motor area activity during mental rotation studied by time-resolved single-trial fMRI. Journal of Cognitive Neuroscience, 12, 310-320.

Rode, G., \& Perenin, M. T. (1994). Temporary remission of representational hemineglect through vestibular stimulation. NeuroReport, 5, 869-872.

Rode, G., Perenin, M. T., \& Boisson, D. (1995). Neglect of the representational space: Demonstration by mental evocation of the map of France. Revue Neurologique, 151, 161-164.

Rubens, A. B. (1995). Caloric stimulation and unilateral visual neglect. Neurology, 35, 1019-1024.
Schwarz, D. W. F., \& Fredrickson, J. M. (1971). Rhesus monkey vestibular cortex: A bimodal primary projection field. Science, 172, 280-281.

Shuren, J., Harley, T., \& Heilman, K. M. (1998). The effects of rotation on spatial attention. Neuropsychiatry, Neuropsychology, and Behavioral Neurology, 11, 72-75.

Snodgrass, J. G., \& Vanderwart, M. (1980). A standardized set of 260 pictures: Norms for name agreement, image agreement, familiarity and visual complexity. Journal of Experimental Psychology: Human Learning and Memory, 6, 174-215.

Storrie-Baker, H. J., Segalowitz, S. J., Black, S. E., McLean, J. A., \& Sullivan, N. (1997). Improvement of hemispatial neglect with coldwater calorics: An electrophysiological test of the arousal hypothesis of neglect. Journal of the International Neuropsychological Society, 3, 394-402.

Suzuki, M., Kitano, H., Ito, R., Kitanishi, T., Yazawa, Y., Ogawa, T., et al. (2001). Cortical and subcortical vestibular response to caloric stimulation detected by functional magnetic resonance imaging. Cognitive Brain Research, 12, 441-449.

Thompson, W. L., \& Kosslyn, S. M. (2000). Neural systems activated during visual mental imagery: A review and meta-analyses. In A. W. Toga \& J. C. Mazziotta (Eds.), Brain mapping II: The systems (pp. 535-560). San Diego: Academic Press.

Tiecks, F. P., Planck, J., Haberl, R. L., \& Brandt, T. (1996). Reduction in posterior cerebral artery blood flow velocity during caloric vestibular stimulation. Journal of Cerebral Blood Flow Metabolism, 16, 1379-1382.

Tong, F. (2003). Primary visual cortex and visual awareness. Nature Reviews Neuroscience, 4, 219-229.

Van Essen, D. C. (1985). Functional organization of primate visual cortex. In A. Peters \& E. G. Jones (Eds.), Cerebral cortex (pp. 259-329). NY: Plenum.

Wallace, B., \& Hofelich, B. G. (1992). Process generalization and the prediction of performance on mental imagery tasks. Memory \& Cognition, 20, 695-704.

Wenzel, R., Bartenstein, P., Dieterich, M., Danek, A., Weindl, A., Minoshima, S., et al. (1996). Deactivation of human visual cortex during involuntary ocular oscillations: A PET activation study. Brain, 119, $101-110$. 